\title{
Development of Mobile Face Verification Based on Locally Normalized Gabor Wavelets
}

\author{
Fadhlan Hafizhelmi Kamaru Zaman *, Ahmad Asari Sulaiman”, Ihsan Mohd Yassin\#, Nooritawati Md \\ Tahir" ${ }^{\#}$ Zairi Ismael Rizman ${ }^{\&}$ \\ "Department of Computer Engineering, Faculty of Electrical Engineering, Universiti Teknologi MARA, Shah Alam, Selangor, Malaysia \\ E-mail:fadhlan@salam.uitm.edu.my,ihsan.yassin@gmail.com,pgrhribu@salam.uitm.edu.my
}

${ }^{\#}$ Department of Communications Engineering, Faculty of Electrical Engineering, Universiti Teknologi MARA, Shah Alam, Selangor, Malaysia E-mail: asari100@gmail.com

${ }^{\&}$ Faculty of Electrical Engineering, Universiti Teknologi MARA, Dungun, Terengganu, Malaysia E-mail:zairi576@tganu.uitm.edu.my

\begin{abstract}
In this paper, we present a mobile face verification framework for automated attendance monitoring as a solution for more efficient, portable and cost-effective attendance monitoring systems. We use Raspberry Pi as mobile embedded input module connecting the webcam and radio frequency identification (RFID) reader to the personal computer (PC) which provides mobility due to its light weight and wireless connectivity. In order to increase the reliability of the system, we incorporate a face verification method which employs locally-normalized Gabor Wavelets as the features for dual verification stage. We evaluate the accuracy and processing time of the proposed face verification. It found that it produces good accuracy under limited reference sample constraint and fast response for a small number of gallery images. The proposed method delivers $97 \%, 99.8 \%$ and $95.3 \%$ accuracy for AR, YALE B and FERET datasets. In term of processing speed, the proposed method managed to classify a single image against 500 gallery images in 1.909 seconds. The system delivers fast verification with high accuracy under the constraint of just single reference sample, which increases the reliability of the proposed system.
\end{abstract}

Keywords - face recognition; Gabor wavelets; local approach; single sample; verification system

\section{INTRODUCTION}

In Universiti Teknologi MARA (UiTM), the process of taking students' attendance still employ a piece of paper where students need to write their signature on the sheet for every class, lab sessions, workshops and even outdoor programs. This method is not reliable since there is a high risk of losing the data. There is also a high possibility of falsification of attendance by the students. Thus it is important to elevate the functionality of attendance management system. Furthermore, a significant increase in a number of the university's students in recent years requires inevitable improvement on the traditional way of monitoring attendance in lectures and students' programs. Additionally, the integrity of students' attendance determined by sole use of matric cards in existing systems such as Easy Access Attendance Management System (EAMS) [1] could be improved further. The main concern regarding EAMS is it is possible that the student can falsely register the attendance on someone's behalf by simply flashing a matric card in front of the scanner. Another issue tackled in this work is most existing attendance systems in the market are not mobile enough to be readily deployed when needed, i.e. for outdoor activities.

Thus, we believe that we can address these problems with an embedded mobile attendance system equipped with dual verification strategy which utilizing both smart card and biometric feature such as face during the registration process for better mobility, accuracy, and reliability. In light of this proposed solution, we are aware that fingerprint, face, and iris are among biometrics properties that are commonly used for person recognition. However, face recognition amassed our interest due to its distinction in accuracy and nonobtrusiveness when used for active person recognition [2], [3]. Additionally, face recognition is the most natural biological features recognition according to the cognitive rule of human beings. Face recognition also possesses the following advantages compared to fingerprint including fast identification, high security, contactless and hygienic. However, the real challenge lies in the limited number of 
reference faces available for a good estimation of the subject identity. In this case, each student may have only single image in university database as a reference. This problem is known Single Sample per Person (SSPP) problem [4]-[6] and one of the solutions for this problem is by using local face recognition approach [7], [8] which is adopted in this work.

We are confident that our proposed solution is better than existing methods in regard to effectiveness and accuracy with dual-verification, required resources and cost as well as in terms of ease of use and deployment. Ultimately, the system can be used not only in UiTM but also in other universities or training centers as well. As a local product, this would clearly benefit the growth of local economy. This solution also can ensure the education in the form of lectures and training are well delivered, and the target audience would fully utilize the opportunity presented to them.

Previously, local strategies have been adopted to overcome the SSPP problems. The strategies involve the partitioning of faces into blocks and subsequently the local patches (LP) are classified using ensembles of classifiers by computing non-metric similarities between LPs of training samples. Martinez has produced three significant works on face recognition under SSPP constraints using probabilistic matching and motion estimation [9]. Besides, in [8] extends local probabilistic approach in Martinez's work using SelfOrganizing Maps (SOM) where they proposed to train a single SOM for all the samples and to train a separate SOM for each class. In addition, in [10] adapts a generic discriminant model to discriminate the persons in SSPP constraints by Adaptive Generic Learning (AGL) method. Recently, Sparsity Preserving Discriminant Analysis (SPDA) is proposed to deal with multi sample [11] and SSPP face recognition [12]. Another recent work in dealing with SSPP problem is a method called Discriminative Multimanifold Analysis (DMMA) [13]. More recently, in [14] proposed another SSPP-based face recognition method called Double Linear Regression (DLR).

On the other hand, local approach alone could not produce good classification result if the features used have poor preservation of spatial locality and possess inadequate discriminative ability. In previous years, Gabor Wavelets (GW) have been identified as one of the best face descriptors for face recognition, and this is largely attributed to GW's biologically relevant kernel that effectively represents facial features [15]-[21]. GW preserves the inherent spatial locality by employing kernel which is identical to the human cortical cells, specifically the receptive field and this preservation of spatial locality is indeed a vital characteristic for an excellent face descriptor. GW features can help preserving optimal intra-class and inter-class separation since the computed features are optimally localized in both space and frequency domains.

Among previous well-known methods of face recognition based on local GW approach are Local Gabor Binary Pattern Histogram Sequence (LGBPHS) [22] and Hierarchical Ensemble Classifier (HEC) [23] where HEC is implemented using a weighted fusion of Local Gabor Feature Vector (LGFV) and global Fourier transform. Additionally, Gabor features are independently classified using ensembles of Borda count in Local Matching Gabor (LMG) method [24].

Recently, LMG is improved using entropy-like weighting strategy and Local Normalization (LN) approach. Gabor wavelets have also been successfully implemented previously for handwritten numeral recognition [25].

\section{MATERIAL AND METHOD}

\section{A. Locally Normalized Gabor Wavelets Features}

In this paper, we adopt the Local Gabor Feature Vector (LGFV) method similar to the implementation of HEC, but with the fusion step dropped and LN process is added to improve the classification accuracy by reducing the effect of illumination variations in image. We use GW to extract features from local square patches of the face image to form a group of feature vectors by combining the features sharing similar spatial information (lateral patches). The images are locally normalized before computing the GW. Thus the proposed method is referred to onwards as LGFV-LN method. GW are computed using Gabor kernel $\psi_{u w}$. Given that $\mathbf{z}$ is the pixel, $z$ is the orientation, $v$ is the scale, $f$ is the step in frequency and $k_{\max }$ is the maximum frequency, which GW can be computed using Equations 1 and 2:

$$
\begin{gathered}
\psi_{u, v}=\frac{\left|h_{u, v}\right|^{2}}{\sigma^{2}} \exp \left(-\frac{\left|h_{u, v}\right|^{2}|z|^{2}}{2 \sigma^{2}}\right)\left[\exp \left(i k_{u, v} \cdot z\right)-\exp \left(-\frac{\sigma^{2}}{2}\right)\right] \\
z=(x, y)^{T}, \quad k_{u v}=\frac{k_{\max }}{f^{v}}\left[\cos \left(\pi \frac{u}{g}\right), \sin \left(\pi \frac{u}{g}\right)\right]^{T}
\end{gathered}
$$

We use 8 orientations $(0 \leq u \leq 7)$ and 5 scales $(0 \leq v \leq 4)$ forming into $40 \mathrm{GW}$ of different scales and orientations. In order to obtain Gabor feature $\mathbf{G}_{\mathrm{ul} v}$ image $\mathbb{I}(\mathrm{z})$ and $\mathrm{GW}$ kernel $\Psi_{u_{w}}$ are convolved such that $\mathbf{G}_{u_{v} v}=\mathbb{I}(\mathbf{z})=\psi_{u_{i} v}$. Since small displacements can linearly affect Gabor phases, we use only the Gabor magnitudes. Thus using Equation 3, we can calculate the magnitude $\mathrm{M}_{\mathrm{u}_{i}, v}$.

$$
M_{u, l}=\sqrt{\operatorname{Im}\left(G_{u_{i}, v}\right)+\operatorname{Re}\left(G_{u_{l},}\right)}
$$

Inspired by previous work, we propose the use of LN image $I_{\mathrm{LN}}(\mathrm{x}, \mathrm{y})$ as the input for the convolution with the Gabor kernel instead of $\mathbf{I}(x, y)$. $\mathrm{I}_{\mathrm{LN}}(\mathrm{x}, \mathrm{y})$ can be obtained from (4) where $\mathbb{I}_{\text {mean }}^{B K a}(x, y)$ denotes the mean of a $3 \times 3$ neighborhood around the pixel $(x, y)$ and $I_{\text {tid }}^{z x a}(x, y)$ is the standard deviation of the $3 \times 3$ neighborhood [26].

Subsequently, we partitioned the acquired Gabor Image (GI) into $\mathrm{m}$ square LPs. The overall process of LGFV-LN features acquisition is illustrated in Fig. 1.

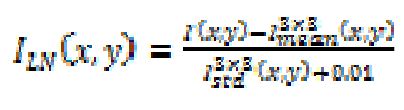



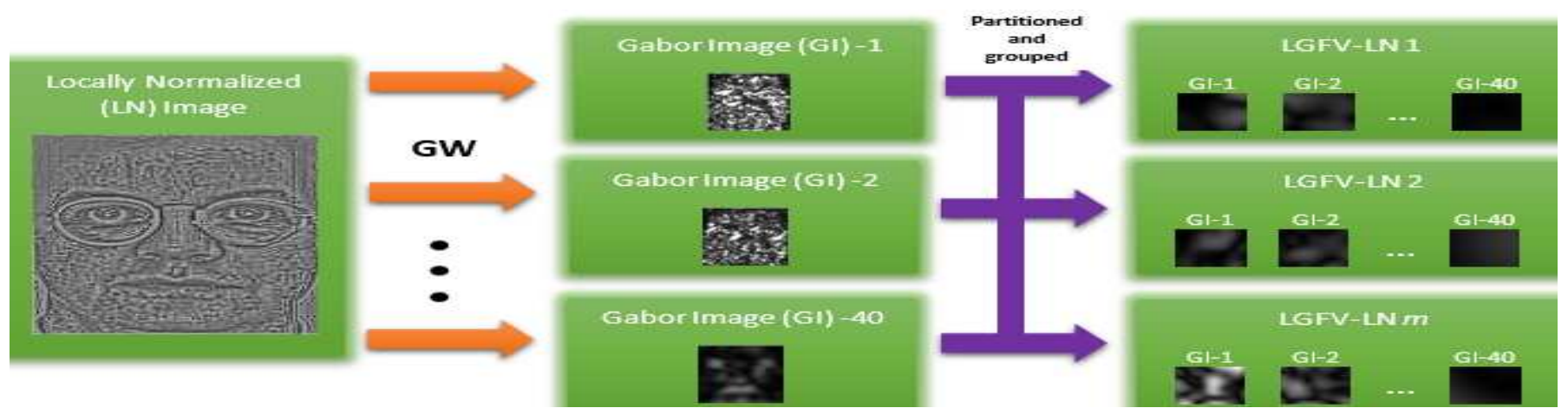

Fig. 1 Overview on process of acquiring LGFV-LN features where each LGFV-LN feature contains 40 spatially grouped LPs from 40 GIs

\section{B. Face Classification}

For face classification, we adopt the local ensemble strategy of k nearest neighbor method called soft $\mathrm{NN}$ where a confidence vector for each local feature was calculated, and then all these confidences were combined by sum aggregation [27]. However, in this work, we use Cosine similarity metrics as opposed to Euclidean distance as some recent works reported good results using Cosine similarity metrics, especially when used with GW features. Given two vectors of attributes $A$ and $B$ where $A_{i}$ and $B_{i}$ are the components of vector $\mathrm{A}$ and $\mathrm{B}$ respectively, the cosine similarity $\mathbf{S}$ can be represented using a dot product and magnitude which given in Equation 5.

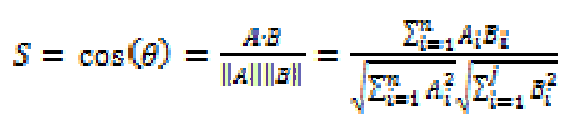

\section{Mobile Face Verification System}

We use Raspberry Pi as mobile embedded input module in the main system framework, where we connect the webcam and RFID reader to the PC wirelessly. This provides the much needed mobility and portability which is due to its light weight and wireless connectivity. Fig. 2 and 3 show the overall framework of the mobile face verification system and the actual implementation of mobile input module respectively. Raspberry $\mathrm{Pi}$ is used to acquire facial image and ID from the person before transmitting the image and ID for further processing at a server PC. In this case, the Raspberry $\mathrm{Pi}$ and the server are connected to the same Access Point (AP).

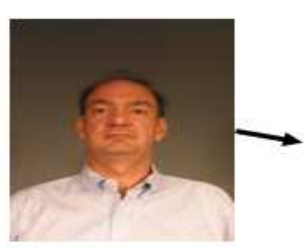

Person

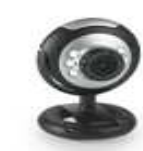
Camera

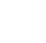

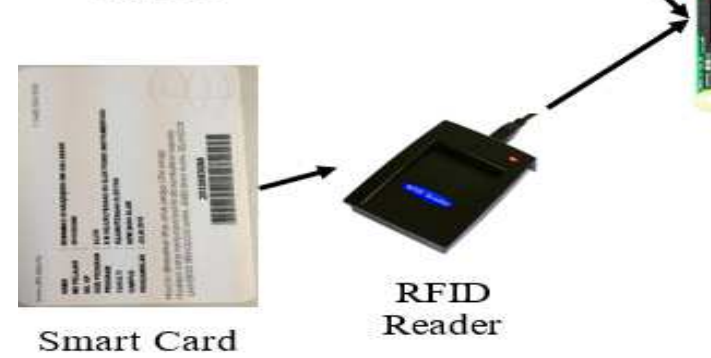

Fig. 2 The proposed framework of the mobile face verification system
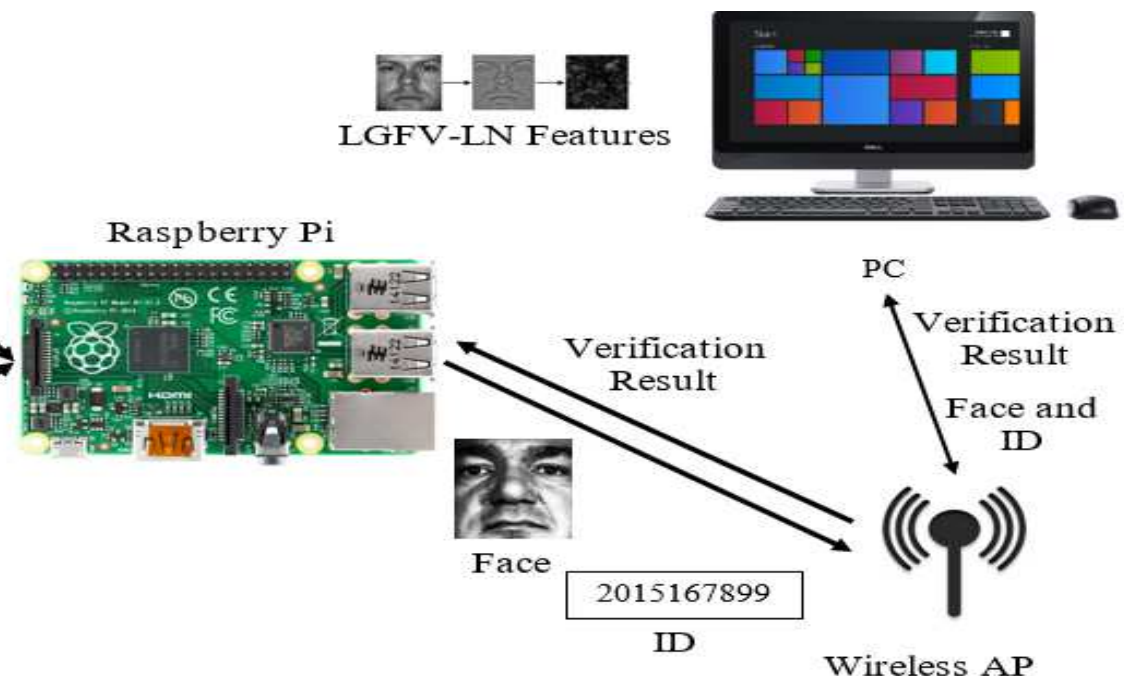

Smart Card

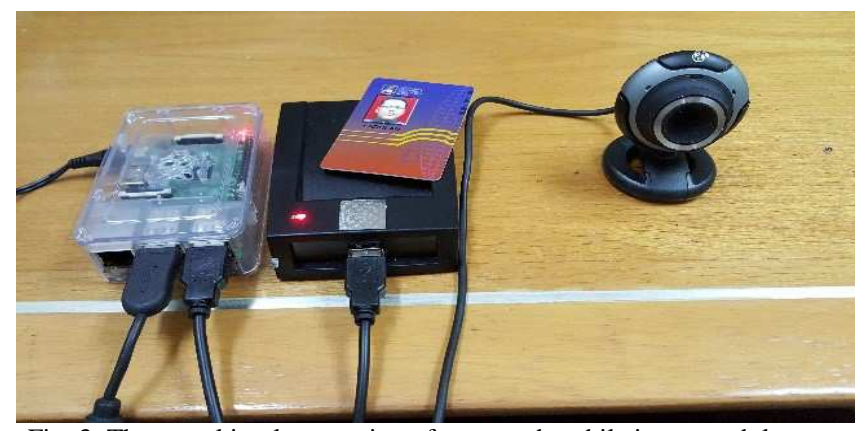

The process of verifying a person is given in Fig. 4 and can be elaborated as follows:

- The subject's ID is acquired as the smart card is flashed on the RFID reader. Subsequently, the subject face is acquired from a webcam connected to the Raspberry Pi using Viola-Jones face detection algorithm [28].

- The acquired face image and ID are then transmitted to a server PC. 
- At the server, the ID is checked against the database entries to verify its existence. If the ID exists, the following procedures continue. Otherwise, the verification returns a negative result.

- The image sent to the server is processed further to obtain LGFV-LN feature. Likewise, the LGFV-LN features for all candidate images in the database are computed beforehand to speed-up the classification process.
- Soft NN classifier is used to find top-n nearest candidates where afterward, the ID for top-n candidates is compared against the subject's ID.

- If one of the top-n candidates' ID matches the subject's ID, the verification returns a positive result. Otherwise, the verification returns a negative result.

- The verification result is sent wirelessly to the Raspberry Pi.

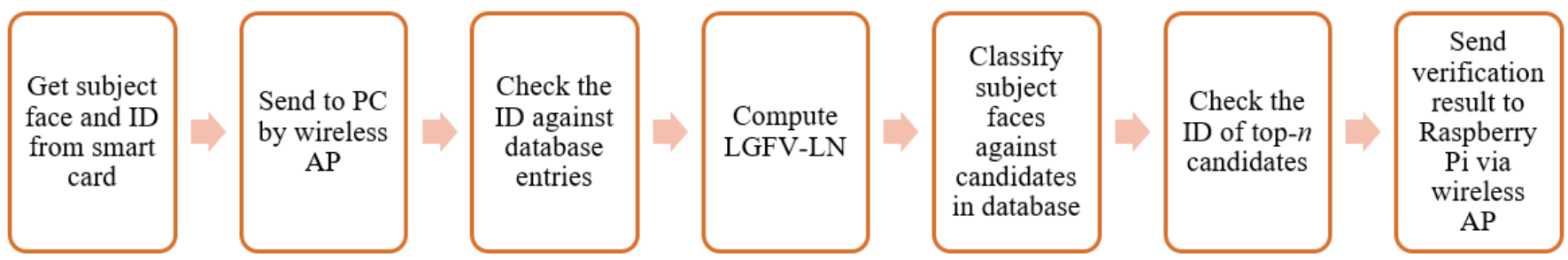

Fig. 4 The process of verifying a person using the proposed mobile face verification system

\section{RESULTS AND DISCUSSION}

For the experiments, three datasets namely AR, YALE B, and FERET are used. The AR dataset [29] contains several types of variations such as different illumination conditions, expressions, and partial occlusions. Out 26 images for each subject, 1 image contain neutral expression (used as gallery image), and the remaining 25 are used as probes-7 images contain expressions, 6 images contain illumination variation, 6 images depict the person as wearing glasses, and the other 6 images depict the person as wearing a scarf. Yale B dataset [30] contains images having different illumination conditions with a wide range of light direction with respect to camera axis defined by azimuth angle $\varphi$ and elevation $\theta$.

38 images having $\varphi=0^{\circ}$ and $\theta=0^{\circ}$ are used as a gallery,

while the other 2376 images having $(\varphi, \theta)>12^{\circ}$ are used

as probe images divided into five subsets. In Figs. 5 and 6 show the images from AR and Yale B datasets respectively. FERET dataset consists of 13,539 facial images corresponding to 1,565 subjects. Following the standard FERET evaluation protocol [31], subset $f a$ containing 1,196 frontal images of 1,196 subjects was used as gallery while $f b$ (1,195 expression-variant images), $f c$ (194 illuminationvariant images), dup I (722 images taken later in time) and $d u p$ II (234 images which are a subset of $d u p$ I) were used as probes. Fig. 7 shows images from FERET dataset.

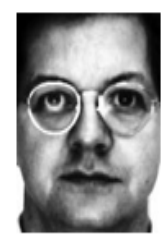

a

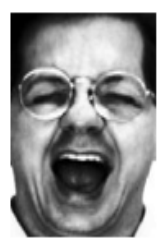

b

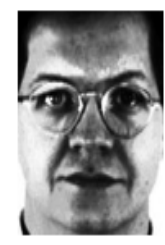

c

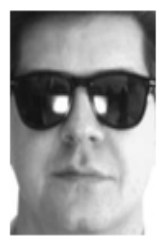

d

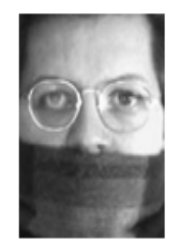

e
Fig. 5 Images in AR dataset consist of (a) neutral expression, (b) expression-variant, (c) illumination (denoted later as ilm.) (d) sunglasses and (e) scarves images

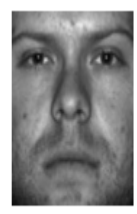

a

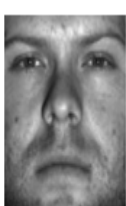

b

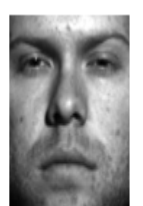

C

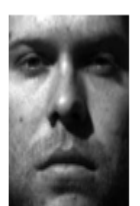

d

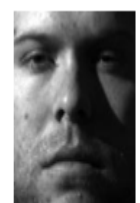

e

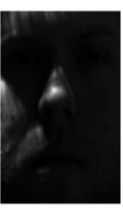

f
Fig. 6 Images in Yale B dataset consist of images with illumination variations, defined by $(\omega, \theta)$, as in (a) $(\varphi, \theta)=0^{\circ}$, (b) $(\phi, \theta)<12$, (c) $12^{\circ} \leq(0, A)<25^{\circ}$, (d) $25^{\circ} \leq(0, \theta) \leq 50^{\circ}$, (e) $50^{\circ} \leq(0, \theta) \leq 77^{\circ}$, (f) $77^{\circ} \leq(0, A)$

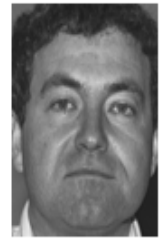

a

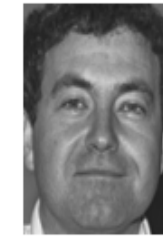

b

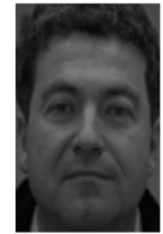

C

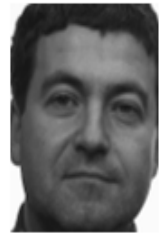

d

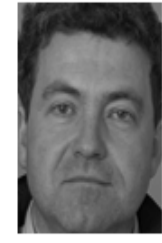

e
Fig. 7 FERET dataset consist of 5 subsets namely (a) fa, (b) fb, (c) fc (d) dupI and (e) dupII

Prior the proposed process, all images used are aligned and resized to $63 \times 63$ pixels, and the intensities of all images

are normalized using LN filter. The values of parameters used in (1) are $0 \leq v \leq 4, \quad 0 \leq u \leq 7$, $f^{\prime}=\sqrt{2} .7, k_{\max }=\pi / 2$, and $\sigma=\pi$. Each Gabor image is then partitioned into $m=81$ square $7 \times 7$ LPs. Unless stated otherwise, all experiments follow SSPP constraints.

\section{A. Results for All Tested Datasets}


Several methods including proposed method are tested with the datasets, and the results are given in Table I. The PI method denotes local face recognition using only image pixel intensities, without application of GW while LGFV method uses local GW approach but without local normalization applied. LN method uses only local normalization without GW applied. Best results in the table are shown in bold.

TABLE I

TOP-1 FACE RECOGNITION ACCURACy FOR All TeSTEd Datasets

\begin{tabular}{|c|c|c|c|c|c|c|c|c|c|c|c|c|c|}
\hline \multirow[t]{3}{*}{ Methods } & \multicolumn{13}{|c|}{ Datasets } \\
\hline & \multicolumn{4}{|c|}{$\mathbf{A R}$} & \multicolumn{5}{|c|}{ YALE B } & \multicolumn{4}{|c|}{ FERET } \\
\hline & exp. & ilm. & glass & scarf & 1 & 2 & 3 & 4 & 5 & fb & fc & dupI & dupII \\
\hline PI & 62.7 & 82.8 & 63 & 58 & 75.1 & 98.4 & 76.9 & 85.7 & 76.6 & 0.1 & 0 & 0.4 & 0 \\
\hline $\mathrm{LN}$ & 63.1 & 79.6 & 40.5 & 52.5 & 94.2 & 98.1 & 86.3 & 64.7 & 85.9 & 0.1 & 0 & 0 & 0 \\
\hline LGFV & 91.1 & 97 & 90.5 & 88.5 & 100 & 100 & 93.6 & 82.2 & 91.6 & 92.7 & 63.9 & 65.7 & 55.5 \\
\hline LGFV-LN & 93.1 & 99.2 & 95.5 & 96.5 & 100 & 100 & 100 & 99.8 & 98.4 & 97.2 & 99.4 & 87.9 & 89.7 \\
\hline
\end{tabular}

Top-1 face recognition accuracy in Table 1 suggests that LGFV-LN delivers the best result for all tested datasets. For a fair comparison, all methods are using Cosine similarity, and it can be observed that the classifier when used with PI and LN fails for $f b, f c$, dupI and dupII. This is due to a problem with the computation of cosine values since the vectors' values were too small. In other datasets, GW features clearly show its descriptive superiority against nonGW methods such as PI and LN. For example, in scarf dataset, LGFV-LN delivers $96.5 \%$ accuracy while PI and LN deliver $58 \%$ and $52.5 \%$ accuracy respectively. Moreover, with the use of local normalization, LGFV-LN manages to produce superior results than LGFV method.

\section{B. Comparison between Cosine Similarity, Euclidean} Distance and Soft SVM for LGFV-LN Classification

Table 2 shows the performance of LGFV-LN with 3 different types of classifiers namely Cosine similarity and

TABLE III

COMPARISON BETWEEN COSINE SIMILARITY, EUCLIDEAN DISTANCE AND SOFT SVM FOR LGFV-LN CLASSIFICATION

\begin{tabular}{|c|c|c|c|c|c|c|c|c|c|c|c|c|c|}
\hline \multirow[t]{3}{*}{ Methods } & \multicolumn{13}{|c|}{ Datasets } \\
\hline & \multicolumn{4}{|c|}{$\mathbf{A R}$} & \multicolumn{5}{|c|}{ YALE B } & \multicolumn{4}{|c|}{ FERET } \\
\hline & exp. & ilm.. & glass & scarf & 1 & 2 & 3 & 4 & 5 & $f b$ & $f c$ & dupI & dupII \\
\hline Euclidean & 89.5 & 96 & 87 & 95 & 100 & 100 & 96 & 92.5 & 91.2 & 97.6 & 94.3 & 76.8 & 74.3 \\
\hline Cosine & 93.1 & 99.2 & 95.5 & 96.5 & 100 & 100 & 100 & 99.8 & 98.4 & 97.2 & 99.4 & 87.9 & 93.1 \\
\hline soft SVM & 83.7 & 96 & 76 & 89 & 100 & 100 & 97.8 & 94 & 93.3 & NA & NA & NA & NA \\
\hline
\end{tabular}

TABLE IIIII

COMPARISON BETWEEN LGFV-LN CLASSIFICATION ACCURACY AND SEVERAL EXISTING METHODS

\begin{tabular}{|l|l|l|l|l|l|}
\hline \multirow{2}{*}{ Methods } & \multicolumn{5}{|c|}{ Datasets } \\
\cline { 2 - 6 } & AR average & $\boldsymbol{f b}$ & $\boldsymbol{f c}$ & $\boldsymbol{d u p I}$ & $\boldsymbol{d u p I I}$ \\
\hline Local Binary Patterns (LBP) [32] & NA & 93 & 51 & 61 & 50 \\
\hline Uniform Pursuit (UP) [33] & 71.9 & NA & NA & NA & NA \\
\hline Local SOM [7] & 88 & 93 & NA & NA & NA \\
\hline DMMA [15] & 79.7 & $\mathbf{9 8 . 1}$ & 98.5 & 81.6 & 83.2 \\
\hline LGFV-LN & $\mathbf{9 5 . 9}$ & 97.2 & $\mathbf{9 9 . 4}$ & $\mathbf{8 7 . 9}$ & $\mathbf{9 3 . 1}$ \\
\hline
\end{tabular}

\section{Top- $n$ Accuracy for $L G F V$ - $L N$}

As a verification system, it is important to consider not only Top-1 candidate but also several other candidates ranked just after the top match. In this case, we show the Top-n accuracy for our proposed LGFV-LN method where $n$ ranges from 1 to 10 . By taking first $n$ matches, we can ensure a higher rate of true positives while keeping false
Euclidean distance for soft $\mathrm{NN}$ and soft SVM, where ensembles of SVM classifiers are used. Based on results in the table, best performances of LGFV-LN are obtained when Cosine similarity is used rather than Euclidean distance or soft SVM. For FERET datasets, soft SVM failed completely since the memory required for computation is too high.

\section{Comparison between LGFV-LN and Existing Methods}

As a benchmark, several existing methods such as Local Binary Patterns (LBP), Uniform Pursuit (UP), Local SOM and DMMA are compared against LGFV-LN. According to Table 3, LGFV-LN delivers highest Top-1 accuracy for all tested datasets except for fb dataset where DMMA produce $98.1 \%$ accuracy which is slightly superior to LGFV-LN's $97.2 \%$ accuracy. Other than that, LGFV-LN demonstrates superiority against all benchmarked methods. positives as low as possible by relying on the descriptive ability of LGFV-LN features. Based on the result in Fig. 8, we can deduce that $n=3$ is suitable for our implementation since it produces optimal performance. For $n=3$, the accuracy for AR, YALE B, and FERET are 97\%, 99.8\%, and $95.3 \%$ respectively. Increasing the value of $n$ further will increase the accuracy slightly, but it will ultimately increase processing time as well as the rate of false positives. 
As for the face verification performance of LGFV-LN, the performance can be illustrated as Receiver Operating Characteristics (ROC) curve for ease of understanding. The performance is measured by plotting the True Positive Rate (TPR) against False Positive Rate (FPR) for PI, LGFV-LN (Cosine) and LGFV-LN (Euclidean). Each dataset used earlier are divided into two parts namely the non-impostors and impostors. The non-impostors are the probes whose reference images were in the gallery, while the impostors are the probes whose reference images were removed from the gallery and random ID were assigned to them. The ROC were obtained by recording the TPR and FPR due to the classification of faces against top-n candidate where $n$ is incrementally changed from 1 to 50 (for YALE B, maximum $\mathrm{n}$ used is 19). The ratio of impostors to non-impostors can be given as 1-to-m ratio, where $\mathrm{m}$ used in this paper is $\mathrm{m}=1$ until $\mathrm{m}=9$. The average of TPR vs. FPR obtained for AR, FERET and YALE B datasets from repeating experiments with different values of $\mathrm{n}$ and $\mathrm{m}$ are shown in Fig. 9.

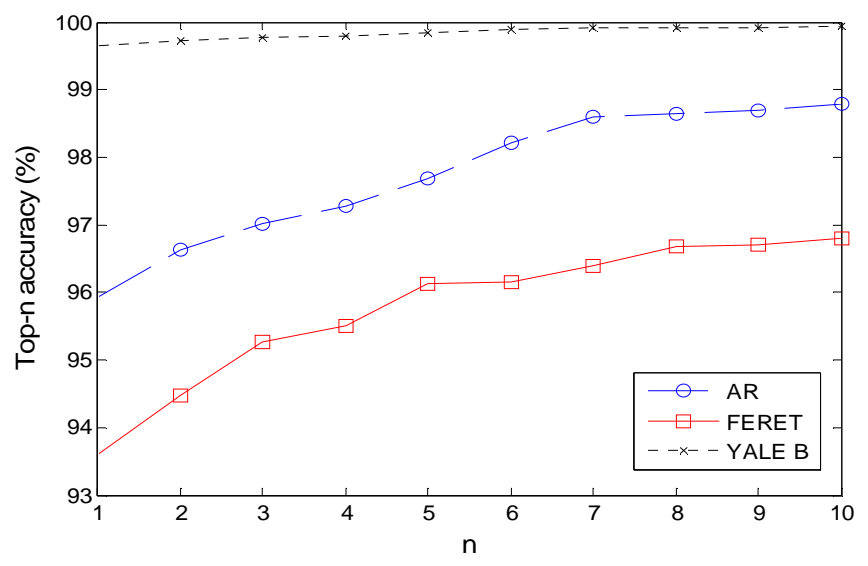

Fig. 8 Top-n accuracy is taken as average accuracy of the corresponding subsets for AR, FERET and YALE B datasets

\section{E. Face Verification Performance for $L G F V$ - $L N$}

Based on Fig. 9, we found that for all tested datasets, LGFV-LN (Cosine) demonstrates superior verification performance against its Euclidean counterpart and PI approach. LGFV-LN (Cosine) is significantly better in terms of maximizing true positives while keeping the false positives minimal. For instance, the (TPR, FPR) performances of LGFV-LN (Cosine) when Top-3 matches are used for AR, FERET and YALE B are (0.970, 0.042), $(0.753,0.004)$ and $(0.998,0.104)$ respectively. For comparison, the corresponding LGFV-LN (Euclidean) method produces $(0.926,0.039),(0.731,0.003)$ and $(0.985$, $0.095)$ respectively while PI method produces $(0.719$, $0.039),(0.573,0.002)$ and $(0.945,0.109)$ respectively. The performance measure in terms of TPR and FPR is very important in face verification system since we want to limit the access that any impostors have. In this case, we wish to reduce the chance of falsification in students' attendance record.

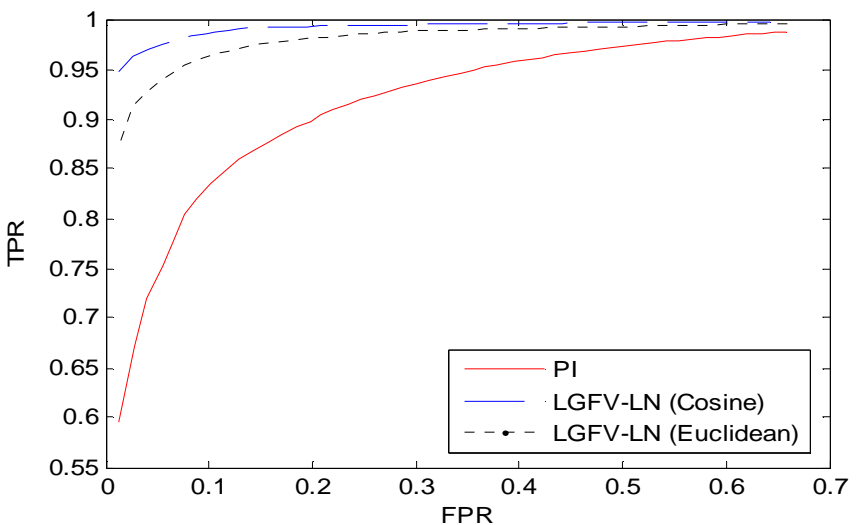

(a)

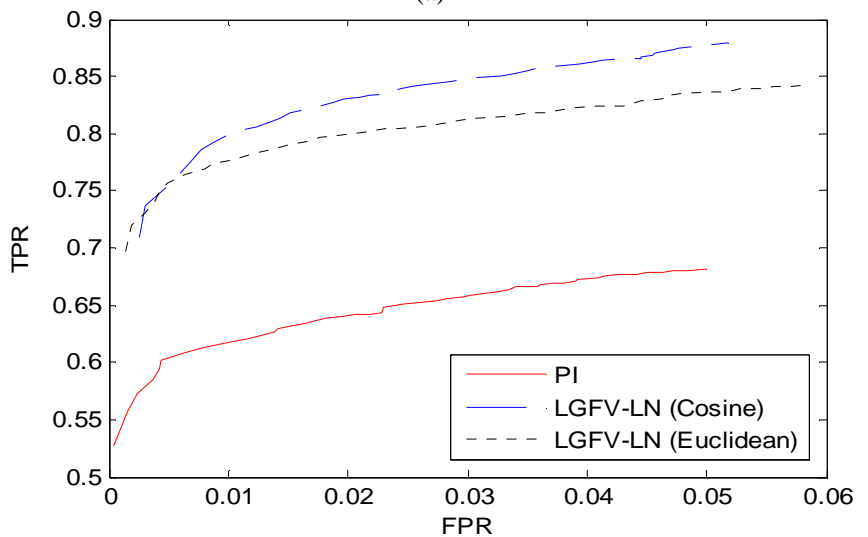

(b)

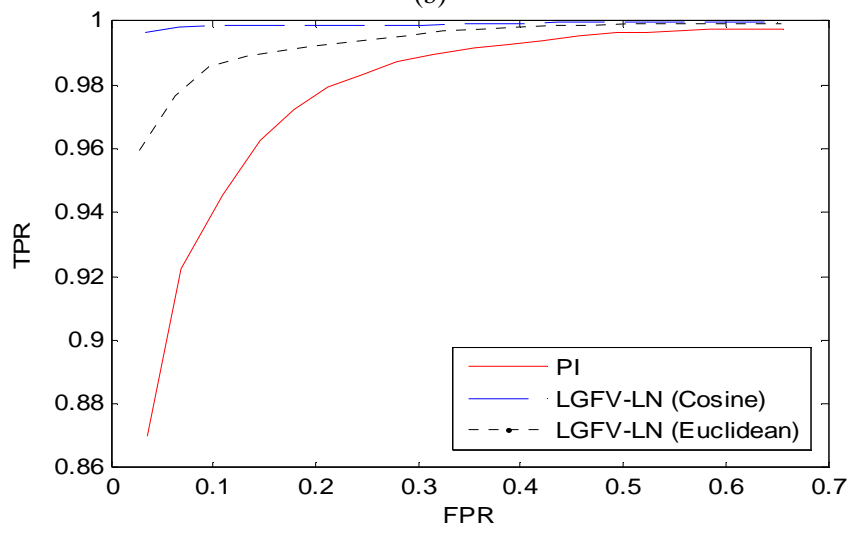

(c)

Fig. 9 Comparisons between ROC curves of face verification carried out using PI, LGFV-LN (Cosine) and LGFV-LN (Euclidean) approaches for several datasets: (a) AR dataset, (b) FERET dataset and (c) YALE B dataset

\section{F. Performance of LGFV-LN in Terms of Processing Speed}

There is a trade-off between the accuracy and speed even though LGFV-LN approach produces higher accuracy than other approaches. To investigate the feasibility of PI, LGFVLN (Cosine) and LGFV-LN (Euclidean) approaches, the processing time required for these methods are examined. This experiment is carried out on a computer running on $3.80 \mathrm{GHz}$ quad-core processor with 4GB RAM. For comparison, the processing time only includes the time required to perform classification. We use one-to-many classification for face recognition, where the face recognition would try to classify single face against a gallery containing multiple images. The result is shown in Fig. 10 for classification of 1 face against up to 1000 gallery images.

Based on result presented in Fig. 10, PI approach is faster than both LGFV-LN approaches where PI only requires only 
0.045 seconds to classify a face against 500 images which is only a fraction of the time required by LGFV-LN (Euclidean) and LGFV-LN (Cosine) which is at 0.888 and 1.909 seconds respectively. Based on this finding, for realtime application, we recommend that LGFV-LN approach is suitable when classifying faces against a small number of gallery images. But for the larger gallery, another approach such as PI would be more suitable. Another factor that would influence the type of suitable approach is the variations in the face to be classified. If local variations are involved, it is better to use LGFV approach. Additionally, if the processing speed is critical, we would recommend using LGFV-LN (Euclidean) approach since it is faster than LGFV-LN (Cosine) but with some sacrifice in accuracy.

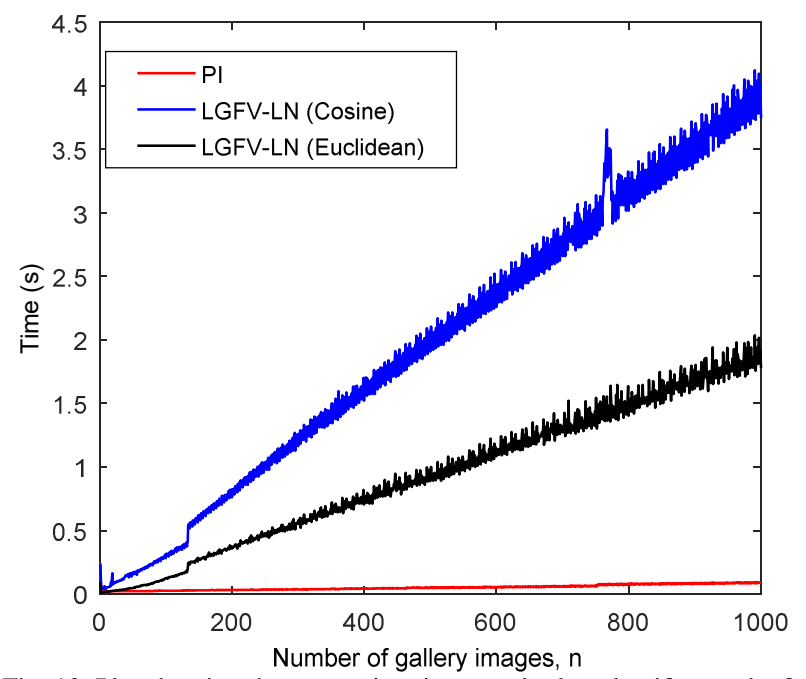

Fig. 10 Plot showing the processing time required to classify a probe face against a gallery containing $\mathrm{n}$ number of images for PI, LGFV-LN (Cosine) and LGFV-LN (Euclidean) approaches

\section{CONCLUSION}

A framework for mobile mobile face verification system which is to be used as attendance monitoring system in UiTM has been proposed in this paper. The face verification method is basedon locally normalized Gabor Wavelets features.. We proposed the use of Raspberry Pi as mobile embedded input module connecting the webcam and RFID reader to the PC wirelessly. We also explained the process of person verification adopted in this system. As for face recognition stage, the proposed Local Gabor Feature Vector with Local Normalization (LGFV-LN) method demonstrates superiority against almost all benchmarked methods in all tested datasets under Single Sample Per Person (SSPP) constraints. The Cosine similarity measure when used with soft NN classifier also delivered the best performance for classification of LGFV-LN features. We also proposed that the verification system to consider up to 3 top matches for optimal performance. The Top-3 accuracy of LGFV-LN (Cosine) for AR, YALE B, and FERET datasets are found to be $97 \%, 99.8 \%$ and $95.3 \%$ respectively. We also found that LGFV-LN (Cosine) produces the best ROC curve, but LGFV-LN approach falls short in processing speed department. As for future work, we can improve the proposed method by systematically reducing the number of LGFV-LN features for better processing speed and accuracy. Additionally, we are going to test whether the proposed method can be implemented to perform the face recognition on the mobile platform, especially on mobile smart phones, rather on a remote $\mathrm{PC}$.

\section{ACKNOWLEDGMENT}

The authors would like to thank Dr Aleix M. Martinez for providing the AR Face Dataset. Portions of the research in this paper use the FERET dataset of facial images collected under the FERET program. The authors would like to thank the FERET Technical Agent, the US National Institute of Standards and Technology (NIST) for providing the FERET dataset. The work presented here is sponsored by Universiti Teknologi MARA, Malaysia under grant 600-RMI/DANA 5/3/LESTARI (20/2015).

\section{REFERENCES}

[1] A. A. Sulaiman, M. A. Bakar, M. Z. Noor, and S. A. Abdullah, "Easy access attendance management system (EAMS)," in Proc. IEEE ICEED'14, 2014, p. 105.

[2] A. K. Jain, "Technology: Biometric recognition," Nature, vol. 449, pp. 38-40, Sep. 2007.

[3] S. Z. Li and A. Jain, Encyclopedia of Biometrics, Heidelberg, Germany: Springer Publishing Company Incorporated, 2015.

[4] A. M. Martínez, "Recognizing expression variant faces from a single sample image per class," in Proc. IEEE CVPR'03, 2003, p. 353.

[5] X. Tan, S. Chen, Z. H. Zhou, and F. Zhang, "Face recognition from a single image per person: A survey," Pattern Recognition, vol. 39, pp. 1725-1745, Sep. 2006.

[6] F. Hafiz, A. A. Shafie, and Y. M. Mustafah, "Face recognition from single sample per person by learning of generic discriminant vectors," Procedia Engineering, vol. 41, pp. 465-472, Dec. 2012.

[7] F. K. Zaman, A. A. Shafie, and Y. M. Mustafah, "Robust face recognition against expressions and partial occlusions," International Journal of Automation and Computing, vol. 13, pp. 319-337, Aug. 2016.

[8] X. Tan, S. Chen, Z. H. Zhou, and F. Zhang, "Recognizing partially occluded, expression variant faces from single training image per person with SOM and soft k-NN ensemble," IEEE Transactions on Neural Networks, vol. 16, pp. 875-886, Jul. 2005.

[9] A. M. Martínez, "Recognizing imprecisely localized, partially occluded, and expression variant faces from a single sample per class," IEEE Transactions on Pattern Analysis and Machine Intelligence, vol. 24, pp. 748-763, Jun. 2002.

[10] Y. Su, S. Shan, X. Chen, and W. Gao, "Adaptive generic learning for face recognition from a single sample per person," in Proc. IEEE CVPR'10, 2010, p. 2699.

[11] L. Qiao, S. Chen, and X. Tan, "Sparsity preserving projections with applications to face recognition," Pattern Recognition, vol. 43, pp. 331-341, Jan. 2010.

[12] L. Qiao, S. Chen, and X. Tan, "Sparsity preserving discriminant analysis for single training image face recognition," Pattern Recognition Letters, vol. 31, pp. 422-429, Apr. 2010.

[13] J. Lu, Y. P. Tan, and G. Wang, "Discriminative multimanifold analysis for face recognition from a single training sample per person," IEEE Transactions on Pattern Analysis and Machine Intelligence, vol. 35, pp. 39-51, Jan. 2013.

[14] F. Yin, L. C. Jiao, F. Shang, L. Xiong, and S. Mao, "Double linear regressions for single labeled image per person face recognition," Pattern Recognition, vol. 47, pp. 1547-1558, Apr. 2014.

[15] J. G. Daugman, "Uncertainty relation for resolution in space, spatial frequency, and orientation optimized by two-dimensional visual cortical filters," JOSA A, vol. 2, pp. 1160-1169, Jul. 1985.

[16] S. M. Zain, S. Sulong, N. M. M. Hashim, and Z. I. Rizman, "Environment for agent-based model in mobile database transaction: A review," ARPN Journal of Engineering and Applied Sciences, vol. 10, pp. 9496-9507, Nov. 2015.

[17] A. Fauzi and Z. I. Rizman, "Design and fabrication of $12 \mathrm{GHz}$ microstrip directional coupler for RF/microwave application," Journal of Engineering Science and Technology, vol. 11, pp. 431442, Mar. 2016.

[18] F. D. M. Fauzi, T. Mulyana, Z. I. Rizman, M. T. Miskon, W. A. K. W. Chek, and M. H. Jusoh, "Supervisory fertigation system using 
interactive graphical supervisory control and data acquisition system," International Journal on Advanced Science, Engineering and Information Technology, vol. 6, pp. 489-494, Aug. 2016.

[19] A. Zabidi, N. M. Tahir, I. M. Yassin, and Z. I. Rizman, "The performance of binary artificial bee colony (BABC) in structure selection of polynomial NARX and NARMAX models," International Journal on Advanced Science, Engineering and Information Technology, vol. 7, pp. 373-379, Apr. 2017.

[20] I. M. Yassin, A. Zabidi, R. Jailani, M. S. A. M. Ali, R. Baharom, A. H. A. Hassan, and Z. I. Rizman, "Comparison between cascade forward and multi-layer perceptron neural networks for NARX functional electrical stimulation (FES)-based muscle model," International Journal on Advanced Science, Engineering and Information Technology, vol. 7, pp. 215-221, Feb. 2017.

[21] R. Abdullah, Z. I. Rizman, N. N. S. N. Dzulkefli, S. I. Ismail, R. Shafie, and M. H. Jusoh, "Design an automatic temperature control system for smart tudung saji using Arduino microcontroller," ARPN Journal of Engineering and Applied Sciences, vol. 11, pp. 95789581, May 2016.

[22] W. Zhang, S. Shan, W. Gao, X. Chen, and H. Zhang, "Local Gabor binary pattern histogram sequence (LGBPHS): A novel nonstatistical model for face representation and recognition," in Proc. IEEE ICCV'05, 2005, p. 786.

[23] Y. Su, S. Shan, X. Chen, and W. Gao, "Hierarchical ensemble of global and local classifiers for face recognition," IEEE Transactions on Image Processing, vol. 18, pp. 1885-1896, Aug. 2009.

[24] J. Zou, Q. Ji, and G. Nagy, "A comparative study of local matching approach for face recognition," IEEE Transactions on Image Processing, vol. 16, pp. 2617-2628, Oct. 2007.

[25] S. Arya, I. Chhabra, and G. S. Lehal, "Recognition of Devnagari numerals using Gabor filter," Indian Journal of Science and Technology," vol. 8, pp. 1-6, Oct. 2015.

[26] X. Xie and K. M. Lam, "An efficient method for face recognition under varying illumination," in Proc. IEEE ISCS'05, 2005, p. 3841.

[27] F. Kamaruzaman and A. A. Shafie, "Recognizing faces with normalized local Gabor features and spiking neuron patterns," Pattern Recognition, vol. 53, pp. 102-115, May 2016.

[28] P. Viola and M. Jones, "Rapid object detection using a boosted cascade of simple features," in Proc. IEEE CVPR'01, 2001, p. 511.

[29] A. R. Martinez and R. Benavente, The AR Face Database, Barcelona, Spain: Computer Vision Center, 1998.

[30] K. C. Lee, J. Ho, and D. J. Kriegman, "Acquiring linear subspaces for face recognition under variable lighting," IEEE Transactions on Pattern Analysis and Machine Intelligence, vol. 27, pp. 684-698, May 2005.

[31] P. J. Phillips, H. Moon, S. A. Rizvi, and P. J. Rauss, "The FERET evaluation methodology for face-recognition algorithms," IEEE Transactions on Pattern Analysis and Machine Intelligence, vol. 22, pp. 1090-1104, Oct. 2000.

[32] T. Ahonen, A. Hadid, and M. Pietikainen, "Face description with local binary patterns: Application to face recognition," IEEE Transactions on Pattern Analysis and Machine Intelligence," vol. 28, pp. 2037-2041, Dec. 2006.

[33] W. Deng, J. Hu, J. Guo, W. Cai, and D. Feng, "Robust, accurate and efficient face recognition from a single training image: A uniform pursuit approach," Pattern Recognition, vol. 43, pp. 1748-1762, May 2010 . 